FUSAV-96/05

MAY 1996

\title{
HIGH ENERGY DENSITY FLUCTUATIONS IN TERMS OF FACTORIAL MOMENTS AND ASSOCIATED FREQUENCY MOMENTSU
}

\author{
Mikuláš Blažek阿 \\ Institute of Physics, Slovak Academy of Sciences, \\ 84228 Bratislava, Slovak Republic
}

\begin{abstract}
We propose to verify relations between quantities which characterize scaling properties of high energy density fluctuations in terms of factorial moments and newly introduced associated frequency moments. Typical examples are presented in frame of systematics developed in the present paper. It involves also several sorts of moments applied so far in the search for intermittency and multifractality. Our approach takes the advantage of relations in which a linear combination of associated frequency moments reduces statistical fluctuations to the same extent as it is done by corresponding factorial moments.
\end{abstract}

\section{Introduction}

It can be taken already as firmly established that several high energy particle density fluctuations reveal multifractality ${ }^{1,2}$. This phenomenon appears quite clearly in lepton, hadron as well as heavy ion induced collisions and it is observed at different energies of colliding objects.

High energy intermittency, or in general, multifractality is usually described in terms of quantities characterizing scaling properties of factorial ${ }^{3,4}, F(q)$, and frequency $^{5,6,7}, G(q)$, moments. From this point of view, the corresponding scaling indices $(a(q)$ and $\tau(q)$, respectively) represent the most frequent quantities one is dealing with in this region of physics.

\footnotetext{
†To be published in Int'l J. Mod. Phys. A

${ }^{a}$ Electronic address: blazek@savba.savba.sk
} 
It is usually argued that in the case of very large multiplicities scaling indices are related quite simply, namely,

$$
a(q)=q-1-\tau(q)
$$

Data available so far show a statistically significant difference between the left and right hand sides of (1), compare Table 1. This circumstance is not surprising because a consistent description of realistic experimental data available so far should be represented by a relation extending (1) which takes into account finite multiplicities.

In the present contribution that extention is given in explicit form, for different definitions of the factorial and associated frequency moments; they are related by the requirement that at very large multiplicities the following type of relation should be satisfied,

$$
F(q)=\omega(q) G(q)
$$

$\omega(q)$ being present due to the appropriate normalization. It will be seen also that for a given finite value of the order $q$ the number of correction terms involved in that extension, is finite.

A short and incomplete survey of the factorial moments (represented by the ratio "numerator over denumerator") which are (or might be) currently applied in high energy physics is seen in Table 2.

Introductory relations mentioned in the next Section give the possibility to include (into the relations between scaling indices) the correction terms appearing due to the finite value of the corresponding multiplicities. In the third Section we discuss first of all the standard approaches while the next Section deals with several other forms of the factorial and associated frequency moments. We emphasize also (in the fifth Section) that there is missed a systematic search for multifractality in high energy physics; so far, in individual cases, there are not known reliable conditions which allow to specify the most appropriate type of statistical moments for the study of multifractality. Conclusions and suggestions are formulated in the last Section.

\section{Preliminaries}

We consider charged particle density distributions in a (pseudo)rapidity window $\Delta Y$; let $n_{m e}$ denote the number of particles observed in the $m$-th bin $(m=1,2, \ldots, M)$ of the $e$-th event $(e=1,2, \ldots, E)$, the equal size of bins being $\delta y=\Delta Y / M$. The non-normalized and non-averaged "primitive" factorial moment is represented by quantity $F\left(n_{m e} ; q\right)$, say, with $q \geq 1$,

$$
\begin{aligned}
F(n ; q) & =n(n-1) \ldots(n-q+1) \\
& =n^{q}+A_{1} n^{q-1}+\ldots+A_{q-1} n \\
& =n^{q}\left[1+\frac{A_{1}(q)}{n}+\ldots+\frac{A_{q-1}(q)}{n^{q-1}}\right]
\end{aligned}
$$

\footnotetext{
${ }^{b}$ It will be seen later that, in general, the term "multiplicity" mentioned at this place, means a quantity which depends on the form of individual moments under consideration.

${ }^{c}$ See the preceding footnote.
} 
where (compare $\left.{ }^{10}\right)$,

$$
\begin{aligned}
& A_{1}=A_{1}(q)=-q(q-1) / 2, \\
& A_{2}=A_{2}(q)=+q(q-1)(q-2)(3 q-1) / 24, \\
& A_{3}=A_{3}(q)=-q^{2}(q-1)^{2}(q-2)(q-3) / 48,
\end{aligned}
$$

etc. and with vanishing $A_{j}(q)$ if $q \leq j, \mathrm{j}=1,2, \ldots$.

In the present paper the assumption is applied that scaling properties identified by several appropriate statistical moments are observed in a range of the number of bins, $M$, say,

$$
M_{\text {low }} \leq M \leq M_{\text {high }}
$$

$M_{\text {high }}$ is usually given by resolution of the devices applied. In high energy physics that quantity is finite (like e.g. also in turbulence where it is known as the Kolmogorov' bound).

We take also as granted that the slopes and intercepts entering the leading order asymptotic terms which characterize the scaling properties of the moments under consideration, are sufficiently accurate so that the next to the leading order terms are much smaller (in absolute value) than the former ones.

Now we show the extension of eq.(1) in several concrete cases.

\section{Standard approaches}

Standard approaches involve following form of factorial and associated frequency moments:

\subsection{Horizontal moments}

If properties of individual events are to be emphasized it is usually expected that the horizontal moments are more appropriate for investigating the scaling properties of a sample of events. In this respect e.g. the muon-proton and muon-deuteron collisions $^{7}$ at $280 \mathrm{GeV}$ as well as the $800 \mathrm{GeV}$ protons interacting with emulsion nuclei ${ }^{8}$ can be mentioned.

The standard horizontal factorial moments, $F_{e}^{(H)}(q)$, characterizing the $e$-th event are introduced by (compare with the case $[\mathrm{X}(\mathrm{A} / \mathrm{C})]$ of Table 2 ; there are quoted also other references),

$$
F_{e}^{(H)}(q)=M^{q-1} \sum_{m=1}^{M} F\left(n_{m e} ; q\right) /\left[N_{e}^{(H)}\right]^{q}
$$

and their vertical averaging gives the full form,

$$
F^{(H)}(q)=\frac{1}{E} \sum_{e=1}^{E} F_{e}^{(H)}(q) .
$$

Denominator of the horizontal moment $(5), N_{e}^{(H)}$,

$$
N_{e}^{(H)}=\sum_{m=1}^{M} n_{m e}
$$

\footnotetext{
${ }^{d}$ To minimize the degree of possible confusion in notation and naming of different moments we write down explicitly all indices involved in respective quantities and summations.
} 
represents the multiplicity of the $e$-th event.

The well known (horizontal) frequency $G$-moments characterizing the $e$-th event,

$$
G_{e}^{(H)}(q)=\left\{\sum_{m=1}^{M} n_{m e}^{q} /\left[\sum_{m=1}^{M} n_{m e}\right]^{q}\right\} \vartheta\left(n_{m e}-q\right)
$$

after vertical averaging lead to

$$
G^{(H)}(q)=\frac{1}{E} \sum_{e=1}^{E} G_{e}^{(H)}(q)
$$

Frequency moments (8) represent the leading order asymptotic term of (5) (up to the normalization) in the limit of very large number of observed particles (a more accurate specification is given below).

Now, we are looking for a relation between the factorial and associated frequency moments just introduced.

Since the low values of multiplicity $n_{m e}, n_{m e}<q$, do not influence the former moments (due to their definition, eq.(5)), the presence of $\vartheta$-function in (8) [with $\vartheta(x)=0$ if $x<0$ and $\vartheta(x)=1$ if $x \geq 0$ ] guarantees the same property of the later ones, eq.(8).

Let us adopt the view that fractal structure is revealed by factorial moments if the following scaling property is observed,

$$
\frac{1}{E} \sum_{e=1}^{E} F_{e}^{(H)}(q) \propto f_{q}^{(H)} M^{a_{q}^{(H)}} .
$$

And the fractal structure is detected by frequency moments if the following scaling property is satisfied,

$$
\frac{1}{E} \sum_{e=1}^{E}\left[N_{e}^{(H)}\right]^{-\lambda} G_{e}^{(H)}(q) \propto\left[N_{0}^{(G),(H)}\right]^{-\lambda} g_{q}^{(H)} M^{-\tau_{q}^{(H)}} .
$$

In eq.(11), $N_{0}^{(G),(H)}$ represents the effective average multiplicity; $N_{e}^{(H)}$ is given by (7), and $\lambda=0,1,2, \ldots$; with $\lambda=0$ the usual form of the scaling property is obtained (as it is applied in ${ }^{5,6}$ and in many other papers). With respect to eq.(3),

$$
F_{e}^{(H)}(q)=M^{q-1}\left\{G_{e}^{(H)}(q)+\frac{A_{1}(q)}{N_{e}^{(H)}} G_{e}^{(H)}(q-1)+\cdots+\frac{A_{q-1}(q)}{\left[N_{e}^{(H)}\right]^{q-1}} G_{e}^{(H)}(1)\right\}
$$

and we conclude that the asymptotic equality (of the form (2)) between moments characterizing a given event,

$$
F_{e}^{(H)}(q)=M^{q-1} G_{e}^{(H)}(q),
$$

is satisfied as far as the (total) multiplicity of that event, $N_{e}^{(H)}$, is very large,

$$
N_{e}^{(H)} \gg 1
$$


The series expansion on r.h.s. of (12) retains finite number of terms; this means that such a linear combination of the frequency $G$-moments reduces statistical fluctuations (contained in all individual frequency moments) to the same extent as it is done by the corresponding factorial moment appearing on the l.h.s. of (12).

Averaging eq.(12) over events,

$$
\begin{aligned}
\frac{1}{E} \sum_{e=1}^{E} F_{e}^{(H)}(q) & =M^{q-1}\left\{\frac{1}{E} \sum_{e=1}^{E} G_{e}^{(H)}(q)+\frac{A_{1}}{E} \sum_{e=1}^{E}\left[N_{e}^{(H)}\right]^{-1} G_{e}^{(H)}(q-1)+\cdots\right. \\
& \left.+\frac{A_{q-1}}{E} \sum_{e=1}^{E}\left[N_{e}^{(H)}\right]^{-q+1} G_{e}^{(H)}(1)\right\}
\end{aligned}
$$

and application of the asymptotics (10) and (11) leads to the resulte,

$$
\begin{aligned}
f_{q}^{(H)} M^{a_{q}^{(H)}-q+1} & =g_{q}^{(H)} M^{-\tau_{q}^{(H)}}+\frac{A_{1}}{N_{0}^{(G),(H)}} g_{q-1} M^{-\tau_{q-1}^{(H)}}+\cdots \\
& +\frac{A_{q-1}}{\left[N_{0}^{(G),(H)}\right]^{q-1}} g_{1}^{(H)} M^{-\tau_{1}^{(H)}} .
\end{aligned}
$$

Equation (16) allows to conclude that the asymptotics (1) is satisfied as far as

$$
N_{0}^{(G),(H)} \gg 1
$$

Moreover, with $q=2$ eq.(16) gives历,

$$
f_{2}^{(H)} M_{2}^{a_{2}^{(H)}-1}=g_{2}^{(H)} M^{-\tau_{2}^{(H)}}-\frac{g_{1}^{(H)}}{N_{0}^{(G),(H)}} M^{-\tau_{1}^{(H)}}
$$

(where $A_{1}(2)=-1$ is applied) and with $q=3$ eq.(16) and (18) lead to

$$
f_{3}^{(H)} M^{a_{3}^{(H)}-2}+\frac{2 f_{2}^{(H)}}{N_{0}^{(G),(H)}} M^{a_{2}^{(H)}-1}=g_{3}^{(H)} M^{-\tau_{3}^{(H)}}-\frac{g_{2}^{(H)}}{N_{0}^{(G),(H)}} M^{-\tau_{2}^{(H)}} .
$$

As far as experimental data allow to extract the value of quantities

$$
\left\{f_{2}^{(H)}, a_{2}^{(H)} ; g_{2}^{(H)}, \tau_{2}^{(H)} ; N_{0}^{(G),(H)}\right\}
$$

eq.(18) allows to verify a consistency relation between them. Then eq.(19) represents a relation between $\left\{f_{3}^{(H)}, a_{3}^{(H)} ; g_{3}^{(H)}, \tau_{3}^{(H)}\right\}$ and the quantities quoted in $(20)$. Analogous conclusion can be formulated if the value $q=4,5, \ldots$ is inserted in (16).

${ }^{e}$ We assume that both asymptotics, (10) and (11), are satisfied simultaneously; similar note is applied also in other cases considered in the present contribution.

${ }^{f}$ In all cases considered in the present paper, factorial as well as associated frequency moments with $q=1$ are equal to unity. With respect to that circumstance the corresponding slopes $\left(a_{1}\right.$ and $\left.\tau_{1}\right)$ identically vanish while the intercepts $\left(f_{1}\right.$ and $\left.g_{1}\right)$ are equal to unity. However, with respect to a better transparency of the corresponding relations, those quantities are not replaced by their numerical value.

${ }^{g}$ The same comment refers also to other cases considered in the present paper. 
Quantities characterizing scaling properties of standard horizontal moments are related by (16); we propose to apply that relation or its special cases (like (18), (19) and other ones obtained with $q=4,5, \ldots)$ for verification of the consistency or for prediction of some of them

Let us note that eq.(12) allows to express an associated frequency moment $G_{e}(q)$ as a linear combination of the factorial moments $F_{e}(q)$ with decreasing order $q$, namely,

$$
G_{e}^{(H)}(q)=\sum_{j=1}^{q}\left\{B(q-j+1) /\left[N_{e}^{(H)}\right]^{q-j+1}\right\} F_{e}^{(H)}(q-j+1) ;
$$

the coefficients $B(q-j+1)$ being expressed in terms of the coefficients $A_{j}(q)$, compare $^{10}$. Now, let us average the aforementioned relation over events and apply the asymptotics $(1 / E) \sum_{e=1}^{E} G_{e}(q) \propto g_{q} M^{-\tau_{q}}$ together with

$$
\frac{1}{E} \sum_{e=1}^{E}\left[N_{e}^{(H)}\right]^{-\lambda} F_{e}^{(H)}(q) \propto\left[N_{0}^{(F),(H)}\right]^{-\lambda} f_{q}^{(H)} M^{a_{q}^{(H)}} .
$$

Comparison of the result which is obtained by this procedure, with eq.(12) leads to the following interesting conclusion,

$$
N_{0}^{(F),(H)}=N_{0}^{(G),(H)} \equiv N_{0}^{(H)}
$$

where $N_{0}^{(G),(H)}$ is introduced by (11); this means that the effective average multiplicity characterizing horizontal factorial moments is the same as that one characterizing associated frequency moments (this conclusion is valid also in other cases treated below).

\subsection{Vertical moments}

If rare events with sharp peaks are not to be missed $^{5}$, the vertical analysis is suggested. In this case the normalized standard vertical factorial moments characterizing a given (say, the $m$-th) bin acquire the form[ (compare [Y(A/C)] of Table 2),

$$
F_{m}^{(V)}(q)=M^{q-1} E^{q-1} \sum_{e=1}^{E} F\left(n_{m e} ; q\right) /\left[N_{m}^{(V)}\right]^{q}
$$

and the consecutive horizontal averaging leads to the full form,

$$
F^{(V)}(q)=\frac{1}{M} \sum_{m=1}^{M} F_{m}^{(V)}(q)
$$

with

$$
N_{m}^{(V)}=\sum_{e=1}^{E} n_{m e}
$$

\footnotetext{
${ }^{h}$ See the preceding footnote.

${ }^{i}$ The factor $M^{q-1}$ guarantees that eq.(1) is satisfied in the corresponding asymptotics.
} 
being the sum of multiplicities which appear in the $m$-th bin of all events. Factorial moments of the form (23), up to the normalization factor, are successfully applied e.g. when there are investigated interactions of $200 \mathrm{~A} \mathrm{GeV}$ sulphur nuclei with gold target $^{11}$, as well as in several other cases (compare e.g. Table 2). The associated vertical frequency moments,

$$
G_{m}^{(V)}(q)=\left\{\sum_{e=1}^{E} n_{m e}^{q} /\left[N_{m}^{(V)}\right]^{q}\right\} \vartheta\left(n_{m e}-q\right)
$$

averaged horizontally, give the full moments

$$
G^{(V)}(q)=\frac{1}{M} \sum_{m=1}^{M} G_{m}^{(V)}(q) .
$$

Now, the fractal structure is observed in terms of the factorial moments if the scaling relation

$$
\frac{1}{M} \sum_{m=1}^{M} F_{m}^{(V)}(q) \propto E^{q-1} f_{q}^{(V)} M^{a_{q}^{(V)}}
$$

is satisfied, and in terms of the associated frequency moments, if it holds,

$$
\frac{1}{M} \sum_{m=1}^{M}\left[N_{m}^{(V)}\right]^{-\lambda} G_{m}^{(V)}(q) \propto\left[N_{0}^{(G),(V)}\right]^{-\lambda} g_{q}^{(V)} M^{-\tau_{q}^{(V)}} .
$$

Application of (3) allows to arrive at the relation,

$$
\begin{aligned}
F_{m}^{(V)}(q) & =M^{q-1} E^{q-1}\left\{G_{m}^{(V)}(q)+\frac{A_{1}}{N_{m}^{(V)}} G_{m}^{(V)}(q-1)+\cdots\right. \\
& \left.+\frac{A_{q-1}}{\left[N_{m}^{(V)}\right]^{q-1}} G_{m}^{(V)}(1)\right\} .
\end{aligned}
$$

It is seen that a simple relation of the form (2) between moments characterizing the $m$-th bin,

$$
F_{m}^{(V)}(q)=M^{q-1} E^{q-1} G_{m}^{(V)}(q)
$$

is satisfied if the multiplicity $N_{m}^{(V)}$, eq.(25), is sufficiently large, i.e.,

$$
N_{m}^{(V)} \gg 1
$$

Averaging (30) over bins and application of the asymptotics (28) and (29) gives,

$$
\begin{aligned}
f_{q}^{(V)} M^{a_{q}^{(V)}-q+1} & =g_{q}^{(V)} M^{-\tau_{q}^{(V)}}+\frac{A_{1}}{N_{0}^{(G),(V)}} g_{q-1}^{(V)} M^{-\tau_{q-1}^{(V)}}+\cdots \\
& +\frac{A_{q-1}}{\left[N_{0}^{(G),(V)}\right]^{q-1}} g_{1}^{(V)} M^{-\tau_{1}^{(V)}} .
\end{aligned}
$$

Now, the asymptotic equation (1) is recovered as far as the effective average multiplicity $N_{0}^{(G),(V)}$ is sufficiently large, i.e.,

$$
N_{0}^{(G),(V)} \gg 1
$$


With $q=2$ and $q=3$, we obtain from (32), respectively,

$$
f_{2}^{(V)} M^{a_{2}^{(V)}-1}=g_{2}^{(V)} M^{-\tau_{2}^{(V)}}-\frac{g_{1}^{(V)}}{N_{0}^{(G),(V)}} M^{-\tau_{1}^{(V)}}
$$

and applying (34),

$$
f_{3}^{(V)} M^{a_{3}^{(V)}-2}+\frac{2}{N_{0}^{(G),(V)}} f_{2}^{(V)} M^{a_{2}^{(V)}-1}=g_{3}^{(V)} M^{-\tau_{3}^{(V)}}-\frac{g_{2}^{(V)}}{N_{0}^{(G),(V)}} M^{-\tau_{2}^{(V)}} .
$$

Equations (34),(35) and other ones obtained with $q=4,5, \ldots$ are analogous to the preceding case (compare with (18) and (19)). Let us add that also in this case, if the

effective average multiplicity $N_{0}^{(F),(V)}$ characterizing the vertical factorial moments, is introduced in an analogous way as in the preceding part (compare with eq.(21)) the following equality is obtained, $N_{0}^{(F),(V)}=N_{0}^{(G),(V)} \equiv N_{0}^{(V)}$.

\subsection{Mixed moments}

Besides the horizontal and vertical analyses also a mixed approach is applied.

(i) Especially, factorial moments of the form (compare with the case $[\mathrm{Z}(\mathrm{A} / \mathrm{C})]$ in Table 2),

$$
\begin{aligned}
F^{(H V)}(q) & =\frac{1}{M} \sum_{m=1}^{M} \frac{\frac{1}{E} \sum_{e=1}^{E} F\left(n_{m e} ; q\right)}{\left[\frac{1}{M} \frac{1}{E} \sum_{e=1}^{E} N_{e}^{(H)}\right]^{q}} \\
& =M^{q-1} E^{q-1} \frac{\sum_{m=1}^{M} \sum_{e=1}^{E} F\left(n_{m e} ; q\right)}{\left[N^{(H V)}\right]^{q}}
\end{aligned}
$$

are applied for instance in ${ }^{12}$ when intermittency in muon-proton scattering at 280 $\mathrm{GeV}$ is investigated. The associated G-function is now expressed in the following form,

$$
G^{(H V)}(q)=\frac{\sum_{m=1}^{M} \sum_{e=1}^{E} n_{m e}^{q}}{\left[N^{(H V)}\right]^{q}} \vartheta\left(n_{m e}-q\right)
$$

$N^{(H V)}$ in (36) and (37) being the total number of charged particles observed in the sample of $E$-events,

$$
N^{(H V)}=\sum_{m=1}^{M} \sum_{e=1}^{E} n_{m e}
$$

and the number of all charged particles in the $e$-th event, $N_{e}^{(H)}$, entering (36) is given by (7).

If the corresponding fractal structure is present in the data, validity of the following scaling properties is expected,

$$
F^{(H V)}(q) \propto E^{q-1} f_{q}^{(H V)} M^{a_{q}^{(H V)}}
$$

and

$$
G^{(H V)}(q) \propto g_{q}^{(H V)} M^{-\tau_{q}^{(H V)}}
$$


In this case, application of (3) leads to the following relation between factorial (36) and associated frequency (37) moments,

$$
\begin{aligned}
F^{(H V)}(q)= & M^{q-1} E^{q-1}\left\{G^{(H V)}(q)+\frac{A_{1}}{N^{(H V)}} G^{(H V)}(q-1)+\cdots\right. \\
& \left.+\frac{A_{q-1}}{\left[N^{(H V)}\right]^{q-1}} G^{(H V)}(1)\right\} .
\end{aligned}
$$

In the asymptotics (39) and (40), eq.(41) gives,

$$
f_{q}^{(H V)} M^{a_{q}^{(H V)}-q+1}=X_{q}^{(H V)}
$$

where

$$
\begin{aligned}
X_{q}^{(H V)} \equiv g_{q}^{(H V)} M^{-\tau_{q}^{(H V)}}+ & \frac{A_{1}}{N^{(H V)}} g_{q-1}^{(H V)} M^{-\tau_{q-1}^{(H V)}}+\cdots \\
& +\frac{A_{q-1}}{\left[N^{(H V)}\right]^{q-1}} g_{1}^{(H V)} M^{-\tau_{1}^{(H V)}}
\end{aligned}
$$

Now, eq.(41) is reduced to (2) and eq.(42) to (1) as far as the multiplicity $N^{(H V)}$, rel.(38), satisfies the condition

$$
N^{(H V)} \gg 1
$$

If $q=2$ we obtain from (42),

$$
f_{2}^{(H V)} M^{a_{q}^{(H V)}-1}=g_{2}^{(H V)} M^{-\tau_{2}^{(H V)}}-\frac{g_{1}^{(H V)}}{N^{(H V)}} M^{-\tau_{1}^{(H V)}},
$$

and with $q=3,4, \ldots$ the relations follow which are similar to the preceding cases.

(ii) To reduce the influence of non-flat rapidity distribution varying within the finite bin width $\delta y=\Delta Y / M$, introduction of the following factor was suggested in ${ }^{13}$,

$$
R(q)=\frac{\frac{1}{M} \sum_{m=1}^{M}\left\langle\rho_{m e}\right\rangle^{q}}{\left[\frac{1}{M} \sum_{m=1}^{M}\left\langle\rho_{m e}\right\rangle\right]^{q}}
$$

angular brackets denote averaging over all events and $\rho_{m e}=n_{m e} / \delta y$ means particle density in the $m$-th bin of the $e$-th event, so that $\left\langle\rho_{m e}\right\rangle$ is the value of single particle rapidity distribution. In our notation, the factor (46) can be expressed as it follows,

$$
R(q)=M^{q-1} \frac{\sum_{m=1}^{M}\left[N_{m}^{(V)}\right]^{q}}{\left[N^{(H V)}\right]^{q}}
$$

Now, the reduced factorial moment $F^{\left(r e d^{\prime}\right)}(q)$,

$$
F^{\left(r e d^{\prime}\right)}(q)=\frac{F^{(H V)}(q)}{R(q)}
$$


(numerator being expressed by (36) and denominator by (47)) acquires the form (compare with $\left[\mathrm{Z}\left(\mathrm{A} / \mathrm{D}_{1}\right)\right]$ of Table 2 ),

$$
F^{\left(r e d^{\prime}\right)}(q)=E^{q-1} \frac{\sum_{m=1}^{M} \sum_{e=1}^{E} F\left(n_{m e} ; q\right)}{\sum_{m=1}^{M}\left[N_{m}^{(V)}\right]^{q}}
$$

and the multiplicity $N_{m}^{(V)}$ is given by (25). At this point, let us introduce the factorial moment $F^{(r e d)}(q)$,

$$
F^{(r e d)}(q)=M^{q-1} F^{\left(r e d^{\prime}\right)}(q) .
$$

The frequency moments (37) are associated also with (49) (as well as with (48)),

$$
G^{(r e d)}(q)=\frac{\sum_{m=1}^{M} \sum_{e=1}^{E} n_{m e}^{q}}{\sum_{m=1}^{M}\left[N_{m}^{(V)}\right]^{q}} \vartheta\left(n_{m e}-q\right) .
$$

As it is seen, there is a remarkable difference between the couple $(36)+(37)$ and $(49)+(50)$.

Now, the scaling properties are formulated in the form,

$$
\begin{aligned}
& F^{(r e d)}(q) \quad \propto \quad E^{q-1} f_{q}^{(r e d)} M^{a_{q}^{(r e d)}} \\
& G^{(r e d)}(q) \quad \propto \quad g_{q}^{(r e d)} M^{-\tau_{q}^{(r e d)}} .
\end{aligned}
$$

Applying an analogous procedure as in the preceding case, the relation between factorial and associated frequency moments reads,

$$
\begin{gathered}
F^{(r e d)}(q)=E^{q-1}\left\{G^{(r e d)}(q)+A_{1} \frac{\sum_{m=1}^{M}\left[N_{m}^{(V)}\right]^{q-1}}{\sum_{m=1}^{M}\left[N_{m}^{(V)}\right]^{q}} G^{(r e d)}(q-1)+\cdots\right. \\
\left.+A_{q-1} \frac{\sum_{m=1}^{M} N_{m}^{(V)}}{\sum_{m=1}^{M}\left[N_{m}^{(V)}\right]^{q}} G^{(r e d)}(1)\right\}
\end{gathered}
$$

In this case the factorial moment $F^{(r e d)}(q),(49)$, is reduced (up to the normalization) to the associated frequency moment $G^{(r e d)}(q)$, (50), if the multiplicity $N_{m}^{(V)},(25)$, satisfies the following relation,

$$
\frac{\sum_{m=1}^{M}\left[N_{m}^{(V)}\right]^{q-j}}{\sum_{m=1}^{M}\left[N_{m}^{(V)}\right]^{q}} \ll 1,
$$

with $j=1,2, \ldots, q-1$. The condition (54) differs considerably from (44).

In the asymptotics (51) and (52), eq.(53) gives,

$$
f_{q}^{(r e d)} M^{a_{q}^{(r e d)}-q+1}=X_{q}^{(r e d)}
$$

where

$$
\begin{aligned}
X_{q}^{(r e d)} \equiv g_{q}^{(r e d)} M^{-\tau_{q}^{(r e d)}} & +A_{1} \frac{\sum_{m=1}^{M}\left[N_{m}^{(V)}\right]^{q-1}}{\sum_{m-1}^{M}\left[N_{m}^{(V)}\right]^{q}} g_{q-1}^{(r e d)} M^{-\tau_{q-1}^{(r e d)}}+\cdots \\
& +A_{q-1} \frac{\sum_{m-1}^{M} N_{m}^{(V)}}{\sum_{m=1}^{M}\left[N_{m}^{(V)}\right]^{q}} g_{1}^{(r e d)} M^{-\tau_{1}^{(r e d)}} .
\end{aligned}
$$

${ }^{j}$ See the preceding footnote. 
With $q=2$, eq.(56) leads to,

$$
f_{2}^{(r e d)} M^{a_{2}^{(r e d)}-1}=g_{2}^{(r e d)} M^{-\tau_{2}^{(r e d)}}-\frac{\sum_{m=1}^{M} N_{m}^{(V)}}{\sum_{m=1}^{M}\left[N_{m}^{(V)}\right]^{2}} g_{1}^{(r e d)} M^{-\tau_{1}^{(r e d)}} .
$$

If the condition (54) is satisfied then (55) with (56) induce relation (1) between scaling indices $a_{q}^{(r e d)}$ and $\tau_{q}^{(r e d)}$.

\section{Some non-standard cases}

To demonstrate the existence of somehow more involved situations we mention at least two following cases:

\subsection{Collisions of lead nuclei with nuclear target}

Study of $158 \mathrm{GeV} / \mathrm{n}$ lead collisions with nuclear target published in $^{14}$ applies an interesting form of the factorial moment proposed in ${ }^{15}$ (compare with the case $\left[\mathrm{Z}\left(\mathrm{A} / \mathrm{G}_{1}\right)\right]$ of Table 2$)$,

$$
F^{(x 1)}(q)=M^{q-1} E^{q-1} \frac{\sum_{m=1}^{M} \sum_{e=1}^{E} F\left(n_{m e} ; q\right)}{\sum_{m=1}^{M} F\left(N_{m}^{(V)} ; q\right)} .
$$

With respect to $(3)$, where the function $F\left(n_{m e} ; q\right)$ is introduced, the moment (58) can be expressed as it follows,

$$
F^{(x 1)}(q)=\frac{M^{q-1} E^{q-1}}{V^{(M)}(q)} \frac{\sum_{m=1}^{M} \sum_{e=1}^{E} F\left(n_{m e} ; q\right)}{\sum_{m=1}^{M}\left[N_{m}^{(V))}\right]^{q}}
$$

or, with respect to (49),

$$
F^{(x 1)}(q)=\frac{1}{V^{(M)}(q)} F^{(r e d)}(q)
$$

In both expresions just mentioned,

$$
V^{(M)}(q) \equiv 1+A_{1} \frac{\sum_{m=1}^{M}\left[N_{m}^{(V)}\right]^{q-1}}{\sum_{m=1}^{M}\left[N_{m}^{(V)}\right]^{q}}+\cdots+A_{q-1} \frac{\sum_{m=1}^{M} N_{m}^{(V)}}{\sum_{m=1}^{M}\left[N_{m}^{(V)}\right]^{q}} .
$$

The frequency moments $G^{(x 1)}(q)$ associated with (60) (as well as e.g. in the case $\left[\mathrm{Z}\left(\mathrm{A} / \mathrm{D}_{1}\right)\right]$ of Table 2$)$ acquire the form of eq.(50), especially',

$$
G^{(x 1)}(q)=G^{(r e d)}(q) .
$$

Now, by means of (59) and (62) we obtain,

$$
\begin{gathered}
F^{(x 1)}(q)=\frac{M^{q-1} E^{q-1}}{V^{(M)}(q)}\left\{G^{(x 1)}(q)+A_{1} \frac{\sum_{m=1}^{M}\left[N_{m}^{(V)}\right]^{q-1}}{\sum_{m=1}^{M}\left[N_{m}^{(V)}\right]^{q}} G^{(x 1)}(q-1)+\cdots\right. \\
\left.+A_{q-1} \frac{\sum_{m=1}^{M} N_{m}^{(V)}}{\sum_{m=1}^{M}\left[N_{m}^{(V)}\right]^{q}} G^{(x 1)}(1)\right\} .
\end{gathered}
$$

\footnotetext{
${ }^{k}$ It is not surprising that different forms of factorial moments lead to the same form of the leading order asymptotic term.
} 
The appropriate scaling properties are formulated in the following way't,

$$
\begin{aligned}
& F^{(x 1)}(q) \propto E^{q-1} f_{q}^{(x 1)} M^{a_{q}^{(x 1)}}, \\
& G^{(x 1)}(q) \propto g_{q}^{(x 1)} M^{-\tau_{q}^{(x 1)}}
\end{aligned}
$$

(bearing in mind eq.(59)).

In this case, eq.(63) allows to obtain

$$
f_{q}^{(x 1)} M^{a_{q}^{(x 1)}-q+1}=\frac{1}{V^{(M)}(q)} X^{(r e d)}(q),
$$

$X^{(r e d)}(q) \equiv X_{q}^{(r e d)}$ being given by (56). If the multiplicities $N_{m}^{(V)}$ are such that condition (54) is valid then (a) eq.(66) reveals that the scaling indices introduced by (64) and (65) satisfy rel.(1), and (b) eq.(63) leads to the asymptotic relation (2). Applying a similar procedure like in preceeding cases, the following relation between lowest order scaling characteristics is obtained from (66) with $q=2$,

$$
f_{2}^{(x 1)} M^{a_{2}^{(x 1)}-1}=\frac{g_{2}^{(x 1)} M^{-\tau_{2}^{(x 1)}}-\frac{\sum_{m=1}^{M} N_{m}^{(V)}}{\sum_{m=1}^{M}\left[N_{m}^{(V)}\right]^{2}} g_{1}^{(x 1)} M^{-\tau_{1}^{(x 1)}}}{1-\frac{\sum_{m=1}^{M} N_{m}^{(V)}}{\sum_{m=1}^{M}\left[N_{m}^{(V)}\right]^{2}}} .
$$

\subsection{Carbon-cooper interactions}

The factorial moments ${ }^{m}$ suggested essentially in ${ }^{15}$ and applied in the intermittency analysis of the carbon-cooper collisions at $4.5 \mathrm{~A} \mathrm{GeV} / \mathrm{c} \mathrm{in}^{16}$ (compare with $[\mathrm{Y}(\mathrm{A} / \mathrm{E})]$ of Table 2),

$$
F_{m}^{(x 2)}(q)=M^{q-1} E^{q-1} \frac{\sum_{e=1}^{E} F\left(n_{m e} ; q\right)}{F\left(N_{m}^{(V)} ; q\right)}
$$

can be expressed in the form

$$
F_{m}^{(x 2)}(q)=\frac{1}{V\left(N_{m}^{(V)} ; q\right)} F_{m}^{(V)}(q)
$$

where the factorial moments $F_{m}^{(V)}(q)$ characterize the standard vertical case, (23), and

$$
\begin{aligned}
V\left(N_{m}^{(V)} ; q\right) & =1+\frac{A_{1}}{N_{m}^{(V)}}+\cdots+\frac{A_{q-1}}{\left[N_{m}^{(V)}\right]^{q-1}} \\
& \equiv \frac{F\left(N_{m}^{(V)} ; q\right)}{\left[N_{m}^{(V)}\right]^{q}}
\end{aligned}
$$

the bin multiplicity $N_{m}^{(V)}$ is given by $(25)$.

\footnotetext{
${ }^{l}$ The fact that factor $V^{(M)}(q)$ entering eq.(60) depends on the number of bins, $M$, prevents us to identify, in general, the intercepts $f_{q}^{(x 1)}$ with $f_{q}^{(r e d)}$ and the slopes $a_{q}^{(x 1)}$ with $a_{q}^{(r e d)}$, as they are introduced by (64) and (51), respectively.

${ }^{m}$ See footnote ${ }^{i}$.
} 
A slight adaptation of (69) and averaging over bins lead to the relation,

$$
\frac{1}{M} \sum_{m=1}^{M} V\left(N_{m}^{(V)} ; q\right) F_{m}^{(x 2)}(q)=\frac{1}{M} \sum_{m=1}^{M} F_{m}^{(V)}(q) .
$$

In the present case, let us expect scaling property of the factorial moments in an extended form, namely,

$$
\frac{1}{M} \sum_{m=1}^{M}\left[N_{m}^{(V)}\right]^{-\lambda} F_{m}^{(x 2)}(q) \propto E^{q-1}\left[N_{0}^{(F)}\right]^{-\lambda} f_{q}^{(x 2)} M^{a_{q}^{(x 2)}}
$$

where $N_{0}^{(F)}$ is the effective average multiplicity characterizing the factorial moments under consideration (and $\lambda=0,1,2, \ldots)$. With respect to the scaling property $(28)$ of the moments $F_{m}^{(V)}(q)$, eq.(68) allows to concluden,

$$
a_{q}^{(x 2)}=a_{q}^{(V)}
$$

and

$$
f_{q}^{(x 2)}\left\{1+\frac{A_{1}}{N_{0}^{(F)}}+\cdots+\frac{A_{q-1}}{\left[N_{0}^{(F)}\right]^{q-1}}\right\}=f_{q}^{(V)} .
$$

Frequency moments $G_{m}^{(x 2)}(q)$ associated with the factorial moments (68), satisfy the relation,

$$
G_{m}^{(x 2)}(q)=\frac{\sum_{e=1}^{E} n_{m e}^{q}}{\left[N_{m}^{(V)}\right]^{q}} \vartheta\left(n_{m e}-q\right)=G_{m}^{(V)}(q),
$$

the last quantity characterizing the standard vertical case, compare eq.(26). Scaling properties of those frequency moments are assumed also in an extended form,

$$
\frac{1}{M} \sum_{m=1}^{M}\left[N_{m}^{(V)}\right]^{-\lambda} G_{m}^{(x 2)}(q) \propto\left[N_{0}^{(G)}\right]^{-\lambda} g_{q}^{(x 2)} M^{-\tau_{q}^{(x 2)}}
$$

where the effective average multiplicity $N_{0}^{(G)}$ specifies scaling properties of the frequency moments under consideration (and $\lambda=0,1,2, \ldots$, as it is usual in this paper).

Relation between moments introduced by (68) and (75) acquires the form,

$$
F_{m}^{(x 2)}(q)=\frac{M^{q-1} E^{q-1}}{V\left(N_{m}^{(V)} ; q\right)} Y_{m}^{(x 2)}(q)
$$

with

$$
Y_{m}^{(x 2)} \equiv G_{m}^{(x 2)}(q)+\frac{A_{1}}{N_{m}^{(V)}} G_{m}^{(x 2)}(q-1)+\cdots+\frac{A_{q-1}}{\left[N_{m}^{(V)}\right]^{q-1}} G_{m}^{(x 2)}(1) .
$$

Let us express (77) in two alternative forms, namely,

$$
V\left(N_{m}^{(V)} ; q\right) F_{m}^{(x 2)}(q)=(M E)^{q-1} Y_{m}^{(x 2)}(q)
$$

\footnotetext{
${ }^{n}$ Compare with observation of ${ }^{17}$ saying that the choice of a different normalization factor does not change the dependence of factorial moments on the number of bins, $M$, as far as this factor is proportional to $M^{q}$.
} 
and

$$
F_{m}^{(x 2)}(q)=(M E)^{q-1}\left[V\left(N_{m}^{(V)} ; q\right)\right]^{-1} Y_{m}^{(x 2)}(q)
$$

where

$$
\left[V\left(N_{m}^{(V)} ; q\right)\right]^{-1}=c_{0}+\frac{c_{1}}{N_{m}^{(V)}}+\frac{c_{2}}{\left[N_{m}^{(V)}\right]^{2}}+\cdots
$$

with $c_{0}=1, c_{1}=-A_{1}, c_{2}=A_{1}^{2}-A_{2}, \ldots$, and $A_{j}$ given by (4). In this case, averaging over bins and application of the scaling properties (72) and (76) in (79) and (80) allow to achieve equality between the effective average multiplicities,

$$
N_{0}^{(F)}=N_{0}^{(G)} \equiv N_{0}^{(x 2)}
$$

Therefore, we obtain from (77),

$$
\begin{aligned}
f_{q}^{(x 2)} M^{a_{q}^{(x 2)}-q+1} & =\frac{1}{V\left(N_{0}^{(x 2)} ; q\right)}\left\{g_{q}^{(x 2)} M^{-\tau_{q}^{(x 2)}}+\frac{A_{1}}{N_{0}^{(x 2)}} g_{q-1}^{(x 2)} M^{-\tau_{q}^{(x 2)}}+\cdots\right. \\
& \left.+\frac{A_{q-1}}{\left[N_{0}^{(x 2)}\right]^{q-1}} g_{1}^{(x 2)} M^{-\tau_{1}^{(x 2)}}\right\}
\end{aligned}
$$

$V(N ; q)$ being given by (70). Especially, with $q=2$,

$$
f_{2}^{(x 2)} M^{a_{2}^{(x 2)}-1}=\frac{g_{2}^{(x 2)} M^{-\tau_{2}^{(x 2)}}-\frac{1}{N_{0}^{(x 2)}} g_{1}^{(x 2)} M^{-\tau_{1}^{(x 2)}}}{1-\frac{1}{N_{0}^{(x 2)}}}
$$

In this case equation (1) between scaling indices is recovered by (83) as far as the effective average multiplicity $N_{0}^{(x 2)}$ is sufficiently large, i.e.,

$$
N_{0}^{(x 2)} \gg 1
$$

while relation of the form (2) between the factorial $F_{m}^{(x 2)}(q)$, eq.(68), and the associate frequency $G_{m}^{(x 2)}(q)$, eq. $(75)$, moments is obtained if the multiplicity $N_{m}^{(V)}$ is very large,

$$
N_{m}^{(V)} \gg 1
$$

as it is also in the standard vertical case, compare eq.(31).

\section{Short survey}

We summarize shortly the main items of the present contribution:

Search for intermittency in high energy data on density fluctuations allows to recognize three sorts of factorial moments which are currently applied (compare columns $X, Y, Z$, in Table 2 and the corresponding references e.g. in line $C$ where some successfull forms can be found).

Concrete formes of factorial moments discussed in the present paper are considered as representative samples of the types included into Table 2, this Table being not complete, several other expressions can be added there. Every form of the factorial moment $F(q)$ can be accompanied by an associateed frequency moment $G(q)$. There exists a relation between (i) the factorial moment $F(q)$ and a linear 
combination of finite number of terms involving the frequency moments $G(q)$ with decreasing order $q$; and (ii) the frequency $G(q)$ moment and a linear combination of finite number of terms involving the factorial moments $F(q)$ of decreasing order $q$.

It is well known ${ }^{3,4}$, that convenient averaging reduces the influence of statistical fluctuations in factorial moments. Therefore, the linear combination of frequency moments just mentioned in case (i) reduces that influence of noise to the same extent as it is done by the corresponding factorial moment. And, on the other hand, a linear combination of the factorial moments mentioned in case (ii) gives rise to the noise of the same level as it is done by the corresponding frequency moment.

Associated frequency moments represent asymptotics of the corresponding factorial moments; in the present contribution, in every case, there are clearly specified the multiplicities which allow to achieve that asymptotics. Let us note that the relation mentioned above in item (i), can be interpreted also in the following way: a finite number of asymptotic (=frequency) moments allows to reconstruct the full (=corresponding factorial) moment. Hence, that relation represents a solution of the appropriate (moments) inverse problem expressed in the form "How to restore the full form of a statistical moment by means of its asymptotics ?" .

Scaling properties of the statistical moments involved are described in terms of slopes (scaling indices), intercepts and if convenient also effective average multiplicities; all those quantities are experimentally accessible. In every case discussed in the present contribution, the aforementioned relation between factorial and linear combination of associated frequency moments is presented also in terms of quantities which characterize those scaling properties. The form of relation which is obtained in this way allows to recover eq.(1) between scaling indices, namely, in the asymptotics also specified in the present contribution. In turn, that relation might serve either as predicting some quantities or as consistency conditions for data to be considered as sufficiently accurate ones.

While the slopes $a(q)$ characterize to a high extent dynamical fluctuations, the slopes $\tau(q)$ can be considered as solutions of extended fundamental equation ${ }^{18,19}$ of the Halsey et al. type?. This circumstance also points out the importance of a link between those two sets of slopes.

\section{Conclusions}

In the present contribution we formulate (exact) relations between quantities which characterize scaling properties of factorial and associated frequency moments. No additional experiment nor any new measurement is needed for verifying those relations by existing data; all information necessary for that purpose is involved in the data presently available .

Systematic analyses of the present as well as the near future data might help to classify the sort of fluctuations $\mathbb{q}^{q}$, as well as their dependence on the type of collision,

${ }^{o}$ That equation can be considered also as a definition of the scaling indices $\tau=\tau(q)$ for arbitrary real value of the order $q$.

${ }^{p}$ Only the data on scaling indices are published usually.

${ }^{q}$ It is understood here that quantities characterizing those fluctuations satisfy relations advocated in the present contribution. Moreover, the fractal phenomena usually reveal their presence only if they are analysed by appropriate quantities; negative results were published, too, concerning e.g. $S+A u \quad$ Ref. $^{20}$, and $O+S$ ion-emulsion ${ }^{21}$ interactions at $200 \mathrm{GeV} / \mathrm{n}$. 
energy, kinematic cuts, etc.

In this region of physics, sufficiently reliable Monte Carlo type simulations are still missing. The present version of the eikonal cascade model ${ }^{22,23} \mathrm{ECCO}$, is less refined $^{2}$ than the more conventional models constructed (in principle) for description of quantities admitting only continuous variations in density distributions. There is a hope that new possibility will be open if there will work the implementation of quantum interference into Monte Carlo generators by modelling the generalized Wigner functions, as it is proposed $\mathrm{in}^{24}$. This hope is amplified by the experience that several kinds of structure can be generated also by modelling the elementary probabilities which govern the branching processes (compare e.g. with ${ }^{25}$ ).

\section{$\mathrm{R}$ e f e r e n c e $\mathrm{s}$}

1. C.P. Singh, Phys. Reports 236, 147 (1993).

2. E.A. DeWolf, I.M. Dremin and W. Kittel, preprint HEN-362, update July 1995.

3. A. Bialas and R. Peschanski, Nucl. Physics B 273, 703 (1986).

4. A. Bialas and R. Peschanski, Nucl. Physics B 308, 857 (1988).

5. R.C. Hwa, Phys. Rev. D 41, 1456 (1990).

6. C.B. Chiu and R.C. Hwa, Phys. Rev. D 43, 100 (1991).

7. I. Derado, R.C. Hwa, G. Jancso and N. Schmitz, Phys. Lett. B 283, 151 (1992).

8. R.K. Shivpuri and V. Anand, Phys. Rev. D 50, 287 (1994).

9. G. Singh, A. Mukhopadhyay and P.L. Jain, Z. Phys. A 345, 305 (1993).

10. M. Blažek, Z. Phys. C 63, 263 (1994).

11. M.I. Adamowich et al., (EMU01 Collab.), Phys. Rev. Lett. 65, 412 (1990).

12. I. Derado, G. Jancso, N. Schmitz and P. Stopa, Z. Phys. C 47, 23 (1990).

13. K. Fialkowski, B. Wosiek and J. Wosiek, Acta Phys. Polonica B 20, 639 (1989).

14. B. Wosiek et al. (KLMM Collab.), In: Proc. XXV-th Int'l Symp. on Multiparticle Dynamics, Stará Lesná, Slovakia, 1995. World Sci., Singapore, in press.

15. K. Kadija and P. Seyboth, Z. Phys. C 61, 465 (1994).

16. E.K. Sarkisyan, L.K. Gelovani, G.L. Gogiberidze and G.G. Taran, Phys. Lett. B 347, 439 (1995).

17. P. Lipa and B. Buschbeck, Phys. Lett. B 223, 465 (1989).

18. T.C. Halsey et al., Phys. Rev. A 33, 1141 (1986). 
19. M. Blažek, Multifractals in High Energy Nuclear Collisions by Solving an Inverse Problem. In: Quantum Inversion Theory and Applications. Ed.: H.V. von Geramb. Springer Verlag, Berlin 1994; p. 389.

M. Blažek, High Energy Nuclear Collisions and Phase Transitions. In: Multiparticle Dynamics 1994. Ed.: A. Giovannini, S. Lupia and R. Ugoccioni. World Sci., Singapore, 1995; p. 189.

20. M.I. Adamowich et al. (EMU01 Collab.), Phys. Rev. Letters 65, 412 (1990).

21. T. Åkeson et al. (Helios Collab.), Phys. Lett. B 252, 303 (1990).

22. R.C. Hwa and J.C. Pan, Phys. Rev. D 45, (1992) 106.

23. R.C. Hwa and J.C. Pan, Phys. Rev. D 46, (1992) 2941.

24. A. Bialas and A. Krzywicki, Phys. Lett. B 354, (1995) 134.

25. M. Blažek, Czech. J. Phys. B 32, (1982) 617.

26. R. Holynski et al., Phys. Rev. Lett. 62, 733 (1989).

27. P. Carruthers, E.M. Friedlander, C.C. Shih and R.M. Weiner, Phys. Lett. B 222, 487 (1989).

28. C.B. Chiu and R.C. Hwa, Phys. Rev. D 45, 2276 (1992).

29. R.C. Hwa and J.C. Pan, Phys. Rev. D 45, 1476 (1992).

30. B. Bushbeck, P. Lipa and R. Peschanski, Phys. Lett. B 215, 788 (1988).

31. F. Botterweck et al. (EHS-NA 22 Collab.), Z. Phys. C 51, 37 (1991).

32. S. Wang et al., Phys. Rev. D 49, 5785 (1994).

33. R. Albrecht et al. (WA 80 Collab.), Phys. Lett. B 221, 427 (1989).

34. A. Bialas and M. Gazdzicki, Phys. Lett. B 252, 483 (1990).

35. E.K. Sarkisyan, L.K. Gelovani, G.G. Taran and G.I. Sakharov, Phys. Lett. B 318, 568 (1993). 


\section{Table captions}

TABLE 1. Experimental slopes $a(q), \tau(q)$ and the expression $\Delta(q) \equiv a(q)+\tau(q)-$ $(q-1)$ characterizing fractal structure of (pseudo)rapidity distributions appearing in three different types of collisions. The quantity $\Delta(q)$ is accompanied by mean quadratic error. The slopes of Ref. ${ }^{7}$ and Ref. $^{8}$ concern the case $[\mathrm{X}(\mathrm{A} / \mathrm{C})]$ of Table 2 while those of Ref. ${ }^{9}$ remind us there the case $[\mathrm{Y}(\mathrm{A} / \mathrm{C})]$.

TABLE 2. Several expressions of numerator and denominator entering definition of the factorial moments which are investigated when searching for scaling properties of high energy density fluctuations. In some references the corresponding moments are involved up to an overall normalization factor. The $q$-th power factorial multinomial is defined by $F(n ; q) \equiv n(n-1) \ldots(n-q+1)$. 
TABLE 1.

\begin{tabular}{|c|c|c|c|}
\hline \multicolumn{4}{|c|}{$\mu-p$ and $\mu-d$ collisions at $280 \mathrm{GeV}$, data from ${ }^{7}$} \\
\hline $\mathrm{q}$ & $a(q)$ & $\tau(q)$ & $\Delta(q)$ \\
\hline 2 & $0.012 \pm 0.002$ & $0.91 \pm 0.01$ & $-0.078 \pm 0.010$ \\
\hline 3 & $0.034 \pm 0.016$ & $1.81 \pm 0.02$ & $-0.156 \pm 0.026$ \\
\hline 4 & $0.12 \pm 0.05$ & $2.64 \pm 0.05$ & $-0.24 \pm 0.07$ \\
\hline 5 & $0.22 \pm 0.11$ & $3.57 \pm 0.12$ & $-0.21 \pm 0.16$ \\
\hline \multicolumn{4}{|c|}{ proton-nucleus int's at $800 \mathrm{GeV} / \mathrm{c}$, data from ${ }^{8}$} \\
\hline $\mathrm{q}$ & $a(q)$ & $\tau(q)$ & $\Delta(q)$ \\
\hline 2 & $0.180 \pm 0.020$ & $0.694 \pm 0.005$ & $-0.126 \pm 0.021$ \\
\hline 3 & $0.484 \pm 0.040$ & $1.269 \pm 0.014$ & $-0.247 \pm 0.042$ \\
\hline 4 & $0.947 \pm 0.050$ & $1.740 \pm 0.024$ & $-0.313 \pm 0.055$ \\
\hline 5 & $1.516 \pm 0.072$ & $2.032 \pm 0.052$ & $-0.452 \pm 0.089$ \\
\hline 6 & $2.035 \pm 0.120$ & $2.493 \pm 0.067$ & $-0.472 \pm 0.137$ \\
\hline \multicolumn{4}{|c|}{${ }^{84} \mathrm{Kr}$ at $1.52 \mathrm{~A} \mathrm{GeV}$ colliding with $E m$, data from ${ }^{9}$} \\
\hline $\mathrm{q}$ & $a(q)$ & $\tau(q)$ & $\Delta(q)$ \\
\hline 2 & $0.023 \pm 0.003$ & $0.645 \pm 0.028$ & $-0.332 \pm 0.028$ \\
\hline 3 & $0.069 \pm 0.008$ & $1.238 \pm 0.058$ & $-0.693 \pm 0.058$ \\
\hline 4 & $0.117 \pm 0.017$ & $1.802 \pm 0.090$ & $-1.081 \pm 0.092$ \\
\hline 5 & $0.148 \pm 0.024$ & $2.347 \pm 0.122$ & $-1.505 \pm 0.124$ \\
\hline
\end{tabular}


TABLE 2.

\begin{tabular}{|c|c|c|c|c|}
\hline & & [Ref.] & [Ref.] & [Ref.] \\
\hline Numerator & A & $\begin{array}{c}\frac{1}{M} \sum_{m=1}^{M} F\left(n_{m e} ; q\right) \\
\text { horizontal } \\
\text { factorial moments }\end{array}$ & $\begin{array}{c}\frac{1}{E} \sum_{e=1}^{E} F\left(n_{m e} ; q\right) \\
\quad \text { vertical } \\
\text { factorial moments }\end{array}$ & $\begin{array}{c}\frac{1}{M E} \sum_{m=1}^{M} \sum_{e=1}^{E} F\left(n_{m e} ; q\right) \\
\text { horizontal+vertical } \\
\text { factorial moments }\end{array}$ \\
\hline Notation & B & $\sum_{m=1}^{M} n_{m e}=N_{e}^{(H)}$ & $\sum_{e=1}^{E} n_{m e}=N_{m}^{(V)}$ & $\sum_{m=1}^{M} \sum_{e=1}^{E} n_{m e}=N^{(H V)}$ \\
\hline $\begin{array}{c}\text { Several } \\
\text { expressions } \\
\text { of } \\
\text { denominator }\end{array}$ & $\begin{array}{l}\mathrm{E} \\
\mathrm{F} \\
\mathrm{G}_{1}\end{array}$ & {$\left[\frac{1}{M} N_{e}^{(H)}\right]^{q} \quad\left[\mathrm{R}^{(H)}\right]$} & {$\left[\frac{1}{E} N_{m}^{(V)}\right]^{q} \quad\left[\mathrm{R}^{(V)}\right]$} & $\begin{array}{c}{\left[\frac{1}{E M} N^{(H V)}\right]^{q} \quad\left[\mathrm{R}^{(H V)}\right]} \\
\sum_{m=1}^{M}\left[N_{m}^{(V)}\right]^{q} \quad\left[\mathrm{R}^{12}\right] \\
\sum_{e=1}^{E}\left[N_{e}^{(H)}\right]^{q} \\
\left(\frac{1}{M E}\right)^{q} F\left(N^{(H V)} ; q\right) \\
\left(\frac{1}{E}\right)^{q} F\left(\frac{1}{M} N^{(H V)} ; q\right) \quad\left[\mathrm{R}^{15}\right] \\
\frac{1}{M} \sum_{m=1}^{M}\left(\frac{1}{E}\right)^{q} F\left(N_{m}^{(V)} ; q\right) \\
\frac{1}{M} \sum_{m=1}^{M}\left(\frac{1}{M}\right)^{q-1} F\left(\frac{1}{E} N_{m}^{(V)} ; q\right)\end{array}$ \\
\hline \begin{tabular}{|c|} 
Full form \\
involves an \\
overall \\
\end{tabular} & $\mathbf{V}$ & vertical averaging & horizontal averaging & \\
\hline \begin{tabular}{|c|} 
Are the \\
effective \\
average \\
multiplicities \\
introduced?
\end{tabular} & $\mathbf{W}$ & yes & yes & no \\
\hline
\end{tabular}

References in line C, $\left[\mathrm{R}^{(H)}\right]$ : Ref. $^{3,4,7,8,11,26,27,28,29}$;

$\left[\mathrm{R}^{(V)}\right]: \quad$ Ref..$^{9,13,23,27,30,31,32} ;$
$\left[\mathrm{R}^{(H V)}\right]:$ Ref. ${ }^{4,12,31,32,33,34,35}$. 